\title{
Observations of flux rope - associated particle bursts with GEOTAIL in the distant tail
}

\author{
A. Belehaki ${ }^{1}$, E. T. Sarris ${ }^{2}$, G. Tsiropoula ${ }^{1}$, R. W. McEntire ${ }^{3}$, S. Kokubun ${ }^{4}$, and T. Yamamoto ${ }^{5}$ \\ ${ }^{1}$ Institute of Space Research, National Observatory of Athens, Metaxa \& Vas. Pavlou Str., Palea Pendeli, GR-152 36, Greece \\ ${ }^{2}$ Demoktritos University of Thrace, Xanthi 67100, Greece \\ ${ }^{3}$ The Johns Hopkins University, Applied Physics Laboratory, Laurel, MD 20723, USA \\ ${ }^{4}$ Solar Terrestrial Environment Laboratory, Nagoya University, Toyokawa Aichi, 442, Japan \\ ${ }^{5}$ Institute of Space and Astronautical Science, Sagamihara, Kanagawa 229, Japan
}

Received: 15 November 1996 / Revised: 30 April 1997 / Accepted: 2 June 1997

\begin{abstract}
Geotail energetic particle, magnetic field data and plasma observations (EPIC, MGF and CPI experiments) have been examined for a number of energetic particle bursts in the distant tail $\left(120 \mathrm{Re}<\left|\mathrm{X}_{\mathrm{GSM}}\right|<\right.$ $130 \mathrm{Re})$, associated with moving magnetic field structures, following substorm onsets. The features obtained from this data analysis are consistent with the distant magnetotail dynamics determined first by ISEE3 observations and explained in terms of the neutral line model. At the onset of the bursts, before plasma sheet entrance, energetic electrons appear as a field-aligned beam flowing in the tailward direction, followed by anisotropic ions. Within the flux rope region, suprathermal ions exhibit a convective anisotropy, which allows determination of the plasma flow velocity, assuming that the anisotropy arises from the Compton-Getting effect. The velocities thus determined in the plasma sheet are estimated to be $200-650 \mathrm{~km} / \mathrm{s}$, and compare favourably with the velocities derived from the CPI electron and proton experiment. The estimated length of magnetic field structures varies between 28 and $56 \mathrm{Re}$ and depends on the strength of the westward electrojet intensification. Finally, the three structures reported here show clear magnetic field signatures of flux rope topology. The existence of a strong magnetic field aligned approximately along the $\mathrm{Y}$-axis and centred on the north-to-south excursion of the field, and the bipolar signature in both $B y$ and/or $B \mathrm{z}$ components, is consistent with the existence of closed field lines extending from Earth and wrapping around the core of the flux rope structure.
\end{abstract}

Key words. Magnetospheric configuration and dynamics $\cdot$ Magnetotail

\section{Introduction}

The most comprehensive and best-supported description of the changes taking place in the magnetotail during substorms is the plasmoid model of Hones $(1976,1977)$. According to this model a neutral line is formed in the near-Earth region of the plasma sheet. Earthward of this neutral line the plasma sheet bulk flow is sunward as newly closed field lines contract toward the Earth. Tailward of the neutral line the flow is accelerated in the antisunward direction in response to Lorentz forces associated with disconnected lobe field lines and largescale plasma pressure gradients. At the point where the last of the closed field lines threading the plasma sheet have undergone reconnection, the rate of reconnection grows "explosively" (Coroniti, 1985) as lobe flux tubes begin to disconnect from the Earth. In the plasmoid model, this is the point where the magnetic flux added to the lobes during the growth phase period, is dissipated. The energy which is liberated as reconnection closes previously open field lines, powers the substorm expansion phase. Continued reconnection at the near-Earth neutral line results in the retreating plasmoid and downstream plasma sheet becoming surrounded by a thickening layer of disconnected lobe field lines, and steady conditions will only be achieved after the plasmoid has departed tailward of the spacecraft location.

A systematic exploration of the distant magnetotail was performed for the first time by the 1983 ISEE3 geotail mission which sampled the Earth's geomagnetic tail to $\sim 238$ Re downtail of Earth. These observations have confirmed the existence of regions of closed-loop field lines which are ejected down the tail following geomagnetic substorm onsets (Hones et al., 1984a, b; Scholer et al., 1984 a,b). The relationship of geomagnetic substorms and plasmoid observations at ISEE3 was investigated by Moldwin and Hughes (1992b; 1993); they concluded that the overwhelming majority of the 
events selected as plasmoids in the ISEE3 deep tail data are related to substorm onset signatures near Earth and, furthermore, that every large isolated substorm produces a plasmoid. According to their survey, the majority (over $84 \%$ ) of plasmoid events follow the substorm onset with a time delay of 5 to $60 \mathrm{~min}$.

Signatures consistent with a passage of plasmoids were found in the deep tail plasma sheet (Hones et al., 1984a, b; Moldwin and Hughes, 1992a; Belehaki et al., 1996a) and also observed in the lobes in the form of compression of the magnetic field (traveling compression regions - TCRs) caused by the bulge in the plasma sheet associated with the plasmoid passing by (Maezawa, 1975; Slavin et al., 1984; Murphy et al., 1987; Moldwin and Hughes, 1992a; Owen et al., 1993; Slavin et al., 1993; Belehaki et al., 1996b; Taguchi et al., 1996).

A detailed analysis of the dispersive onset of energetic particles observed by ISEE3, associated with plasmoid structures, has been presented by Scholer et al. (1984a). At the beginning of these bursts they first observed streaming electrons at the energy range of 75-115 keV, followed after several minutes by anisotropic protons, which exhibited positive energy dispersion. Eight to fifteen minutes after the electron onset, the electron distribution became isotropic. They suggested that the spacecraft encounters detached plasma structures evidenced by isotropic electrons, moving with high velocity $(\sim 800 \mathrm{~km} / \mathrm{s})$ downstream. They concluded that velocity dispersion was caused as the thickening layer of reconnected field lines engulfed the spacecraft. But since they failed to observe gradient anisotropies at the edge of the layer, probably because their time resolution was insufficient to show them clearly, they suggested that velocity dispersion effects may also be due to spatial structures moving across the spacecraft. Since there is a dawn-to-dusk electric field near the reconnection region, energetic particles from the region close to the neutral line will necessarily be layered. This layer drapes around the flux rope and when the structure approaches the spacecraft the layer may be pushed over the spacecraft and this will lead to the observation of time dispersion effects.

Richardson and Cowley (1985) presented results from the study of ion bursts associated with plasmoids using data from the energetic particle anisotropy spectrometer (EPAS) on ISEE3. They concluded that burst onsets are associated with a layer of energetic ions which is swept across the spacecraft predominantly by the downtail passage of the plasmoid. A similar layer is observed at the trailing edge of the event as the plasma sheet thins during substorm recovery and contracts back over the spacecraft. Richardson et al. (1993) made a general survey of the spatial distribution of the electron bursts observed by ISEE3 in the geomagnetic tail and the related plasma electron and magnetic field signatures. The observations suggested that predominantly closed magnetic field lines (associated with trapped electrons) were present in the plasma sheet within $\sim 90 \mathrm{Re}$ downtail of Earth, and predominantly open field lines at greater distances. This magnetic field configuration is consistent with the presence of a neutral line in the deep tail at around $90 \mathrm{Re}$ downtail of Earth, inferred independently from ISEE3 studies of plasma, energetic ion flows and of the magnetic field north south component (Zwickl et al., 1984; Daly et al., 1984; Scholer et al., 1984c; Slavin et al., 1985).

The plasmoid picture is essentially two-dimensional and generalizing this to three dimensions allows for the possibility that flux rope may form. Indeed, Hughes and Sibeck (1987) have shown that the magnetic field topology that results from reconnection at a new nearEarth neutral line when the magnetic field in the plasma sheet has a finite dawn-to-dusk component is very different from the O-type plasmoid model. In the threedimensional model of Hughes and Sibeck (1987), reconnected plasma sheet field lines form a magnetic flux rope-like structure which stretches across the tail, but whose ends are connected to the auroral ionosphere, one end in each hemisphere. Although flux rope signatures are expected to be similar to those of closed-loop plasmoids, Sibeck et al. (1984) pointed out three differences between the observed flux ropes and closed-loop plasmoids. First, the magnetic field strength is as strong in the centre of the flux ropes as in the lobes, whereas weaker fields are expected as the spacecraft samples the interior of a closed loop plasmoid (Stern, 1979). Second, no double peak magnetic field signature had been reported in the direction perpendicular to the plasmoid's bipolar signature, as is seen in the three flux rope events reported in that paper. Finally, no bipolar By signature had been reported for plasmoids, while one of the three flux ropes had this characteristic.

A very detailed analysis of CDAW 8 observations of plasmoid signatures in the geomagnetic tail was presented by Slavin et al. (1989). The ISEE3 magnetic field, plasma and energetic particle measurements taken in two plasmoids have been analyzed and compared with various equilibrium structures and propagating waves/ tail oscillation modes. Their results indicate general consistency with either the closed-loop (Hones, 1977) or very small pitch angle flux rope (Hughes and Sibeck, 1987; Birn et al., 1989) models of plasmoid structure and poorer agreement with the other hypothesis. Furthermore, southward magnetic fields are observed in the plasma sheet Earthward of the plasmoid, as expected, due to continued reconnection of open lobe field lines following the formation of the plasmoid by reconnection primarily of closed plasma sheet field lines. An analysis of the $\mathbf{J} \times \mathbf{B}$ forces exerted by these disconnected field lines on the plasmoid suggests that they are sufficient to produce the accelerations necessary to yield the observed tailward speeds at the correct locations and times relative to substorm onset.

Recently, Lui et al. (1994) have examined six magnetic field structures attributed to flux ropes or plasmoids observed by GEOTAIL in the distant tail and reported asymmetries of energetic ions with respect to the core magnetic field of the structure. A possible interpretation is that these plasma structures are connected to a particle source or sources actively modifying their compositional content while they are being ejected downstream beyond $\sim 100$ Re. 
The goals of this study are: first, to determine the internal magnetic and plasma configuration of largescale magnetic field structures associated with energetic ion bursts, observed by GEOTAIL at $\mathrm{X} \sim-125 \mathrm{Re}$; second, to study the dispersive onset of different energy ranges of ions and energetic electrons at the beginning of the particle bursts and third, to give an estimate of the speed of these structures. By accomplishing these goals a picture of the evolution and development of these structures may also be suggested.

\section{Instrumentation and data sets}

Energetic particle measurements used in this study are from the energetic particles and ion composition (EPIC) experiment (Williams et al., 1994). The instrument performs three-dimensional distribution measurements by using both total energy (LEMS, low energy composition system) and velocity/composition detectors (ICS, ion composition system), measuring ions and electrons with energies $>20 \mathrm{keV}$, and ions with energy $>8 \mathrm{keV} /$ nucleon, respectively. The instrument also measures the non-thermal components to $6 \mathrm{MeV}$ for protons, $480 \mathrm{keV}$ for electrons, and $400 \mathrm{keV} /$ nucleon for ions with $\mathrm{Z}>2$. Directional measurements with a time resolution $<1 \mathrm{~s}$ are possible. For the purposes of this study we used the following ICS channels: E3, E4, E5, M2 and ED1. Table 1 presents an overview of the channel characteristics.

The magnetic field is measured by the fluxgate magnetometer (MGF) on board GEOTAIL (Kokubun et al., 1994). The objective of this experiment is to measure the magnetic field variation of the magnetotail in the frequency below $50 \mathrm{~Hz}$. The MGF experiment consists of dual three-axis fluxgate magnetometers and a three-axis search coil magnetometer. The fluxgate magnetometers operate in seven dynamic ranges to cover various regions of the Earth's magnetosphere and the solar wind, and supply 16 vectors/s. Here, we use spin averages $(3 \mathrm{~s})$ of the original high-time-resolution (16 samples/s) data.

Plasma observations have been obtained from the CPI experiment onboard GEOTAIL, which records three-dimensional velocity distribution functions of electrons and positive ions, with identification of ion species. The instrument contains three sets of quadrispherical analysers which obtain three-dimensional measurements for hot plasma and solar wind electrons, for solar wind ions, and for positive-ion composition measurements (Frank et al., 1994). For the needs of this

Table 1. EPIC/ICS channels

\begin{tabular}{lll}
\hline Channel & Resolution & Description \\
\hline E3 & $6 \mathrm{~s}$ & All ions of $62-74 \mathrm{keV}\left(\right.$ mostly H$\left.^{+}\right)$ \\
E4 & $6 \mathrm{~s}$ & All ions of $74-89 \mathrm{keV}\left(\right.$ mostly H$\left.^{+}\right)$ \\
E5 & $3 \mathrm{~s}$ & All ions of $89-110 \mathrm{keV}\left(\right.$ mostly H$\left.^{+}\right)$ \\
M2 & $6 \mathrm{~s}$ & Oxygen of $187-222 \mathrm{keV}\left(\right.$ mostly O$\left.^{+}\right)$ \\
ED1 & $3 \mathrm{~s}$ & Electrons $>38 \mathrm{keV}$ \\
\hline
\end{tabular}

work we used one-minute averages computed using the sum of the three $20 \mathrm{~s}$ instrument cycles. In the cold flowing plasmas typical of the plasma mantle and the low-latitude boundary layer the hot plasma analyser often does not have sufficient resolution of energies and angles needed to fully resolve the ion streams (Paterson, 1996, personal communication). These intervals are obvious because there is a large discrepancy in the densities of the electrons and ions. However, the ion velocity will generally be accurate to within $15^{\circ}$ in direction and about $20 \%$ in magnitude.

\section{Observations and analysis}

We have studied three isolated electron and ion bursts, associated with a fast moving magnetic field structure, during which GEOTAIL has detected highly anisotropic tailward streaming particles, following an extended period of low particle intensities (background level). The events presented here are typical particle events associated with structured magnetic field, that meet the definition of "plasmoid" as introduced by previous papers of Zwickl et al. (1984), Slavin et al. (1985), Moldwin and Hughes (1992b), and Lepping et al. (1996)

a. The magnetic field showed bipolar signature in GSM $B z$ and/or By component with peak-to-peak amplitude of at least $3 \mathrm{nT}$ and not coincident with a neutral sheet crossing, detected by the change of sign in $B x$ (Moldwin and Hughes, 1992b).

b. Two plasma properties were imposed to ensure that the region of maximum field intensity (Lepping et al., 1996) was in the plasma sheet. Plasma density, $N \ll 1 \mathrm{~cm}^{-3}$ and electron temperature, $T_{e}>6 \times 10^{5} \mathrm{~K}$. As discussed by Zwickl et al. (1984) and Slavin et al. (1985), these criteria will generally exclude the magnetosheath, the tail lobes and the tail boundary layer regions.

\section{a. Event of March 31, 1993 at 0135 UT}

The summary plot of the first event, on March 31, 1993, from 0130 UT to 0215 UT is given in Fig. 1. We present magnetic field data of $3 \mathrm{~s}$ time resolution, together with energetic particle data from the EPIC channels ED1, E5, E3, and M2 from five sectors covering an angle of $112.5^{\circ}$ centred at the Sun (sectors Dawn +2 , Dawn +3 , Sun, Sun +1 , Sun +2 , as indicated on the right-hand-side legend of Fig. 1). At the start of this interval GEOTAIL was located in the south lobe at $(\mathrm{X}, \mathrm{Y}, \mathrm{Z}) \approx(-126.4$, 8.8, 3.7) $\mathrm{R}_{\mathrm{E}}$ in GSM coordinates. Since the tail axis aberrated by the Earth's orbital motion is typically displaced by $\sim 8.5 \mathrm{R}_{\mathrm{E}}$ towards dusk at this downstream distance, GEOTAIL was only $\sim 0.3 \mathrm{R}_{\mathrm{E}}$ from the nominal aberrated tail axis. The magnetic field data show clear signatures of a flux rope structured magnetic field. The bipolar signature is along the Bz component and the core field primarily is aligned along the By component, whereas a smaller core field is measured along the $B \mathrm{x}$ 


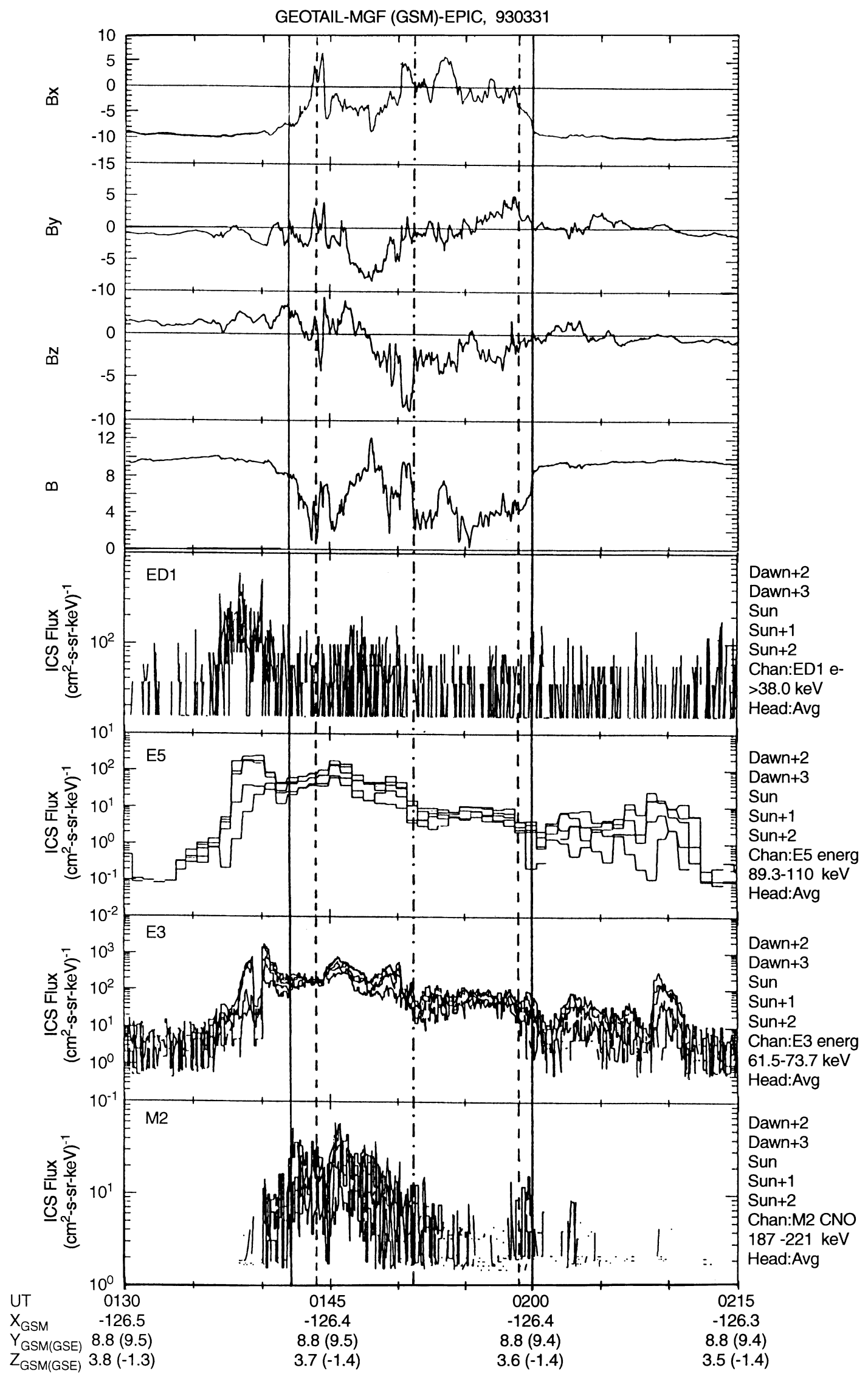



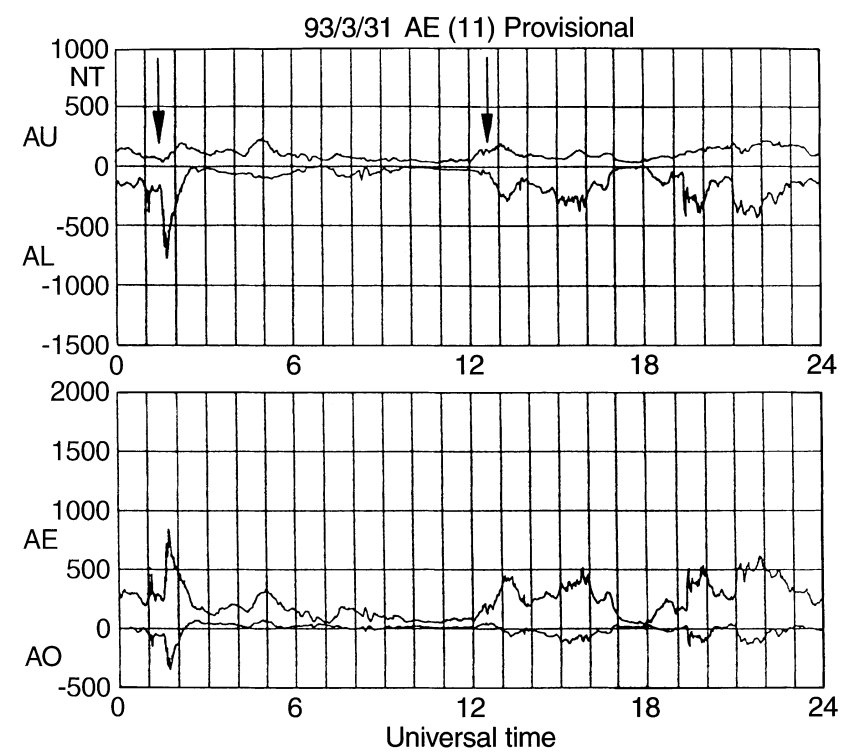

Fig. 2. The AE indices for the day 31 March 1993. The two arrows indicate the onset of the two substorms at 0133 UT and 1242 UT, detected prior to the two flux ropes centred at $\sim 0147$ UT and $\sim 1307$ UT.

component. Note the very strong magnetic field in the core of the structure right at the inflection point of the bipolar signature. This large core field exceeds the previous lobe field magnitude by as much as $25 \%$. Also shown in Fig. 2 is the set of AE indices for that day. Note that the magnetic structure appears at GEOTAIL about $11 \mathrm{~min}$ after the substorm onset, which is marked by the first arrow in Fig. 2. We determine the boundaries of the flux rope using the duration of convected ion population which is the bipolar signature duration plus the time interval during which the spacecraft is located on the post-plasmoid plasma sheet where southward $\mathrm{Bz}$ is observed. To make easier for the reader to follow the successive displacement of GEOTAIL in various magnetospheric regions we mark some important time epochs with vertical lines, in the overview presentation of the event in Fig. 1: the solid vertical lines indicate the two time epochs 0142 UT and 0200 UT, when GEOTAIL leaves the south lobe and returns to it respectively. The two dashed vertical lines indicate the start time

Fig. 1. An overview of the magnetic field and energetic particle conditions around the energetic particle burst on March 31, 1993 at 0137 UT. The top four panels show the magnetic field in GSM coordinates at $3 \mathrm{~s}$ resolution. The bottom 4 panels show the differential energy fluxes of the energetic particle channels ED1, E5, E3 and $M 2$. For each channel we overplotted the fluxes of the sectors looking towards the Sun, with the adjacent sectors, two towards dawn and two dusk, as indicated on the right-hand-side of each panel. The solid vertical lines indicate the two time epochs 0142 UT and 0200 UT, when GEOTAIL leaves the south lobe and returns to it respectively. The two dashed vertical lines indicate the start (0144 UT) and the end times (0159 UT) of convection interval. The start time of the convection interval is coincident with the start of bipolar signature in the $B \mathrm{z}$ component. The time of Earthward edge of the bipolar signature encountered is marked by the dashed-dotted vertical line (0152 UT).
(0144 UT) and the end time (0159 UT) of convection interval. The start time of the convection interval is coincident with the start of bipolar signature in the $\mathrm{Bz}$ component. The time of Earthward edge of the bipolar signature encountered is marked by the dashed-dotted vertical line (0152 UT). Thus, for this event the flux rope interval is from $0144 \mathrm{UT}$ to $0159 \mathrm{UT}$. The exact estimation of these time intervals and important time epochs will be given in the following, using the angular distribution of energetic particles together with the highresolution magnetic field data.

The characteristics of the plasmas are also examined for this flux rope. The key parameters (1-min resolution) of the proton and electron number densities, proton and electron temperatures (solid line for protons and dashed lines for electrons) and the components of proton bulk flow are displayed in order from upper to lower panels in Fig. 3. The number densities are below $1 \mathrm{~cm}^{-3}$ and the electron temperature is greater than $6 \times 10^{5} \mathrm{~K}$; thus, this event meet the selection criteria that exclude the magnetosheath, the lobes and the tail boundary layer regions during the flux rope encounter. The south lobe was encountered during the intervals $0130 \mathrm{UT}$ to

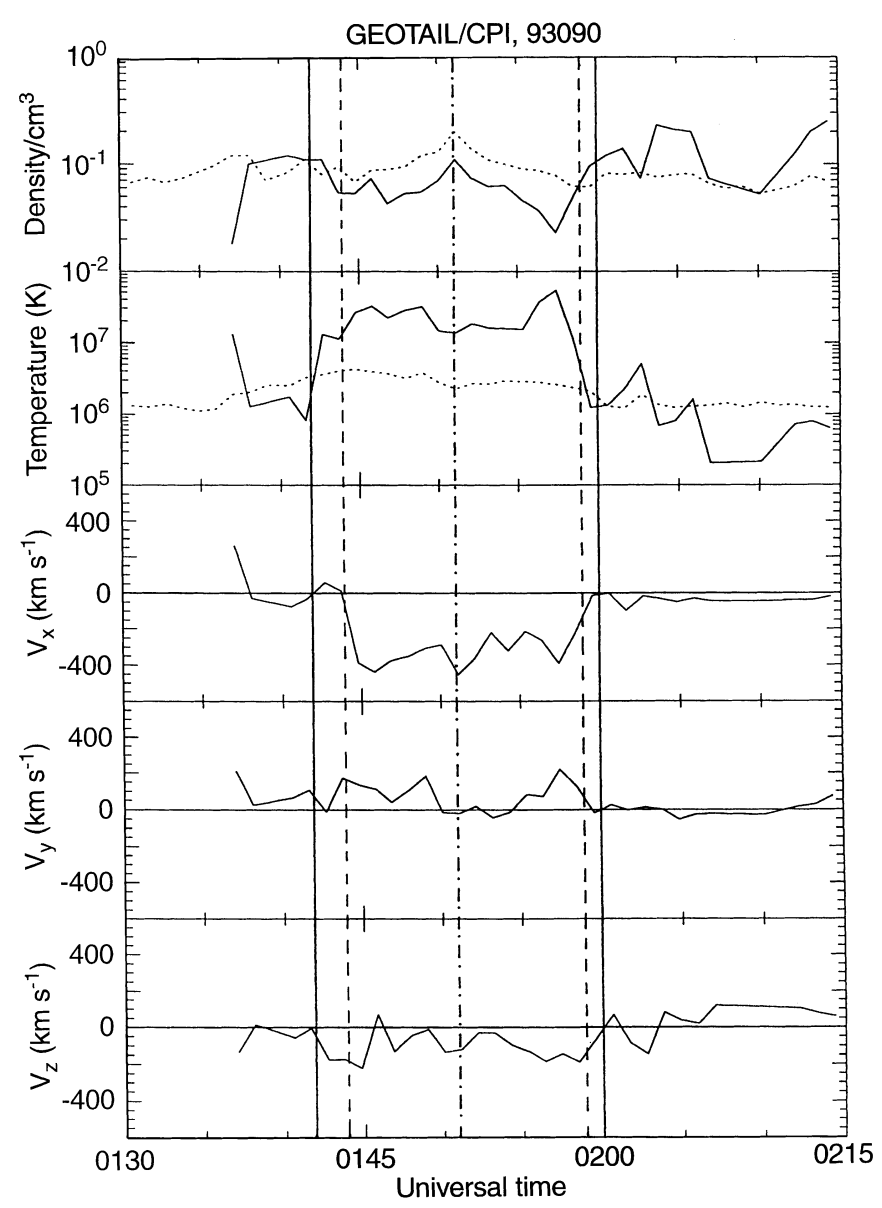

Fig. 3. The KP parameters of number densities and temperatures for protons (solid line) and electrons (dashed line) are shown in the upper two panels for the flux rope signature centred at 0147 UT on March 31,1993 . The components of proton bulk flow are shown in the lower three panels. The vertical lines are plotted as in Fig. 1. 
$0142 \mathrm{UT}$ and $0200 \mathrm{UT}$ to $0215 \mathrm{UT}$. In the lobe the electron and proton temperatures were less than $2 \times 10^{6} \mathrm{~K}$ and the tailward bulk speed was about $50 \mathrm{~km} / \mathrm{s}$ for the most of the duration of these intervals. At $\sim 0145$ UT GEOTAIL is already in the plasma sheet. At that time, the tailward flowing plasmas observed has a bulk speed of $420 \mathrm{~km} / \mathrm{s}$.

In a first look at ICS fluxes we note a sharp enhancement of electrons while GEOTAIL is in the lobe and a rapid decay as GEOTAIL crosses the PSBL and enters the plasma sheet. In contrast the ion intensity remains high for the whole duration of the event, until GEOTAIL re-enters the south lobe after the passage of the magnetic structure.

The angular distributions of energetic electrons and protons are presented in Fig. $4 \mathrm{a}$ and $\mathrm{b}$ respectively. The ion intensity is plotted linearly versus viewing direction and normalized to the sector with maximum intensity. The Sun lies to the right of this figure. The time shown at the top of each plot is the start time of the $6 \mathrm{~s}$ accumulation interval. The number given at the top of each plot is the count rate (counts/s) of the sector with the maximum flux. Together with each angular distribution we overplot the equatorial vector of the mean value of the magnetic field during each $6 \mathrm{~s}$ time interval. The dashed line in each plot is the perpendicular to the field direction. The angular distribution of the energetic electrons with $\mathrm{E}>38 \mathrm{keV}$ are shown in Fig. 4a. Anisotropic electrons above background, are observed from 0136:54 UT to 0139:18 UT. In the tailward anisotropy a cross-tail component is observed in most cases. This anisotropy lasts more than 2 min prior to flux rope arrival at GEOTAIL. After $\sim 0140$ UT, when GEOTAIL is still in the south lobe, the systematic dawn-to-dusk gradient anisotropy of energetic electrons is destroyed.

The angular distributions of $61.5 \mathrm{keV}$ to $73.7 \mathrm{keV}$ ions, mostly protons, are presented in Fig. 4b. At 0136:41 UT we sample the angular distributions of particles in the quiet lobe. Up to 0138:06 UT, a gradient anisotropy perpendicular to the field, which is typical of ions in the lobes near the edge of the PSBL, is generally detected (Cowley et al., 1984; Richardson and Cowley, 1985). While the fluxes are increasing, the ions show positive velocity dispersion (Fig. 1) and strong tailward anisotropy (Fig. 4b, from 0138:42 UT to 0142:43 UT). The particle distributions have also a cross-tail component on the tailward streaming edge which is probably a gradient anisotropy. This can be interpreted as the result of crossing a boundary, as a layer of particles is moving towards the spacecraft. In other words, this can be regarded as the signature of the PSBL displacement across the spacecraft that lasts for about $4 \mathrm{~min}$. At 0143:56 UT the distribution becomes broader and the magnetic field direction presents large deviation from the X-direction. This type of distribution is dominant until 0159:01 UT. The convection interval (0144 to 0159 UT) is marked with the two vertical dashed lines in both Figs. 1 and 3. Within this interval the tailward directed ion flux may be considered as the high energy tail of the thermal ion population convected with the magnetic structure (Richardson et al., 1987). During the convection interval, a north-to-south turning of the $\mathrm{Bz}$ component is observed from 0144 UT to $0151 \mathrm{UT}$, while from $0151 \mathrm{UT}$ until the end of convection that is at 0159 UT, a southward magnetic field is observed in the post-plasmoid plasma sheet Earthward of magnetic structure, as predicted by the reconnection model of substorms. After flux rope passage at 0200:00 UT GEOTAIL still detects tailward streaming ions for several minutes as it is seen from the last two plots presented in Fig. 4b. The spacecraft has re-entered the PSBL, as can also be seen in Fig. 1. The sequence of events during this flux rope associated particle event detected by GEOTAIL is summarized in Table 2.

The convective (bulk) speed of the suprathermal particle population after 0144 UT can be estimated indirectly from the Compton-Getting effect (Gold et al., 1975). The computed bulk velocity varies between $500 \mathrm{~km} / \mathrm{s}$ and $900 \mathrm{~km} / \mathrm{s}$ during the convection period (from 0144 UT to 0152 UT). From measurements of the spectral index $(\gamma \sim 2.8)$ and the anisotropy ratio (the forward versus the backward count rate $\mathbf{J}_{\mathrm{f}} / \mathbf{J}_{\mathrm{b}}=8$ ) at the time of the onset, the Compton-Getting transformation gives a bulk speed $u_{\text {bulk }} \approx 620 \mathrm{~km} / \mathrm{s}$, which is in good agreement with the average plasma sheet velocity observed in situ with GEOTAIL/CPI experiment. Given that the high energy tail of the thermal ion population convected with the magnetic structure is observed for about $15 \mathrm{~min}$, the length of the magnetic structure based upon a mean convection speed from the plasma analyser of $\sim(400 \pm 80) \mathrm{km} / \mathrm{s}$ is $\sim(56 \pm 11) \mathrm{Re}$.

\section{b. Event of March 31, 1993 at 1245 UT}

During the second event under study, GEOTAIL was located at geocentric solar - magnetospheric co-ordinates $\mathrm{X}=-124 \mathrm{Re}, \mathrm{Y}=7.8 \mathrm{Re}$ and $\mathrm{Z}=0.4 \mathrm{Re}$. The overview of this flux rope associated particle burst detected on March 31, 1993 between 1245 UT and 1320 UT is presented in Fig. 5. The first four panels
Table 2. The sequence of events as determined from the analysis of GEOTAIL data for the flux rope signature centred at 0147 UT on March 31, 1993.

\begin{tabular}{|c|c|c|}
\hline Time & Observation & GEOTAIL position \\
\hline 0133 UT & Substorm onset on the ground & South lobe \\
\hline 0137 UT & Tailward streaming electrons & South lobe \\
\hline 0137 UT to 0143 UT & Velocity dispersion of energetic particles & PSBL \\
\hline 0144 UT to 0159 UT & Convection & \\
\hline - 0144 UT to $0151 \mathrm{UT}$ & - Bipolar Bz & - Plasma sheet \\
\hline • 0151 UT to $0159 \mathrm{UT}$ & - Southward Bz & - Post-plasmoid plasma sheet \\
\hline
\end{tabular}




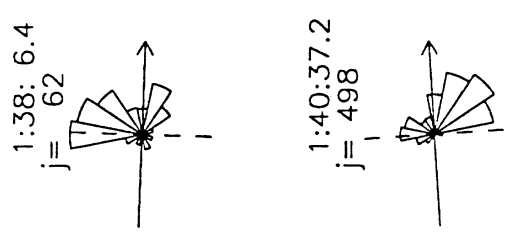

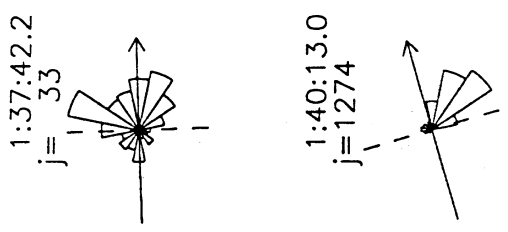

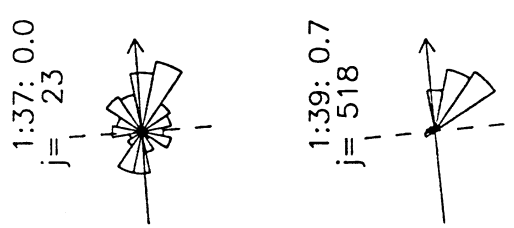

它

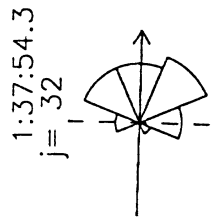

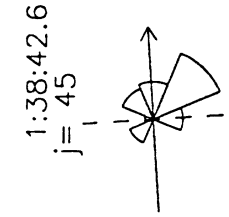

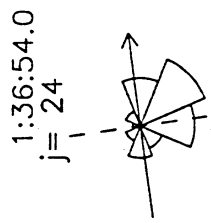

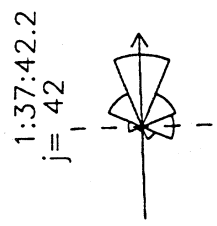

官

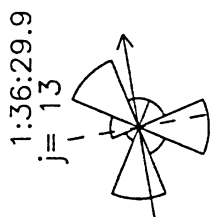

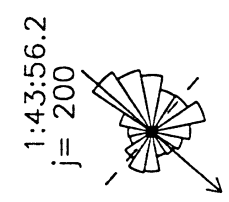

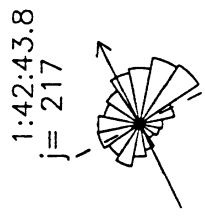

(1)

몬
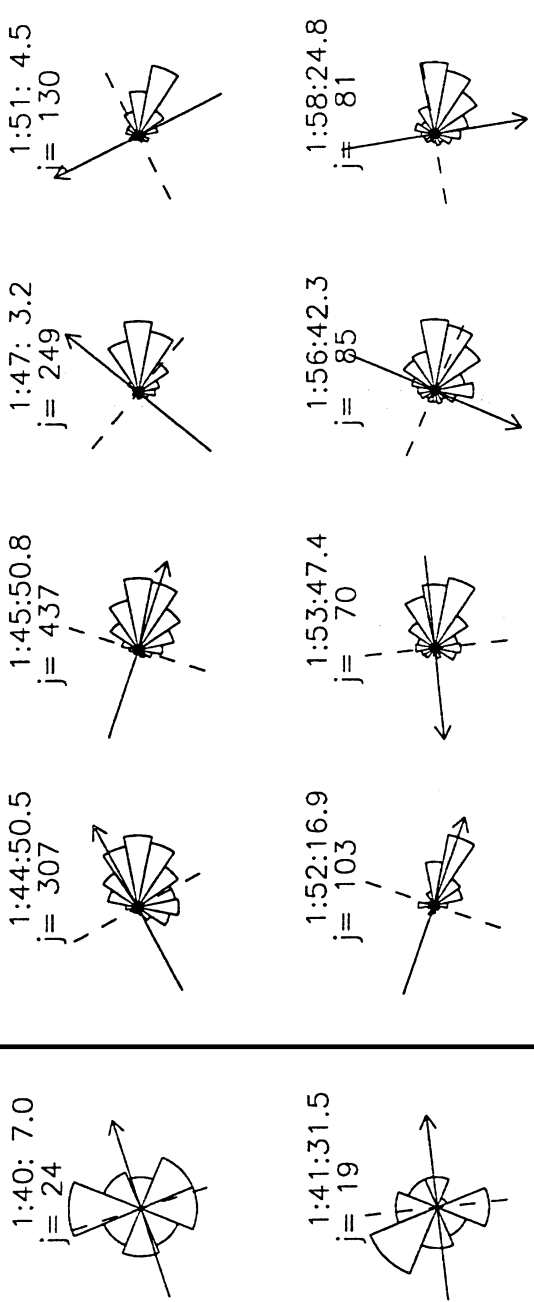

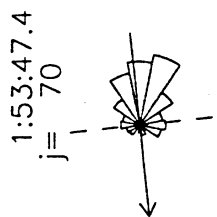

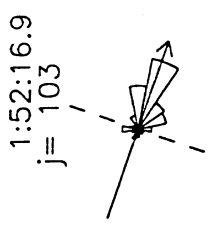

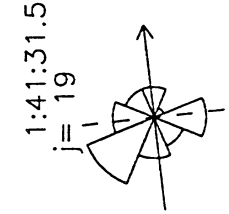

品

$\stackrel{\infty}{\circ} \stackrel{\infty}{\circ}$

$\stackrel{m}{\dddot{m}}$

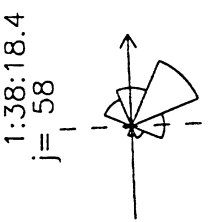

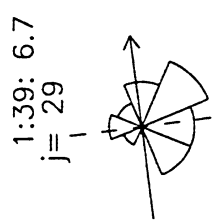

文

$\stackrel{+}{\ddot{\infty}} \stackrel{\sim}{\ddot{m}-1}$
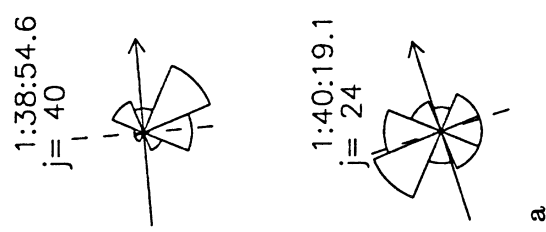

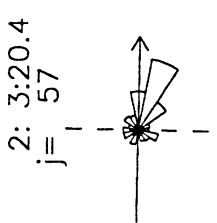

号

กั.

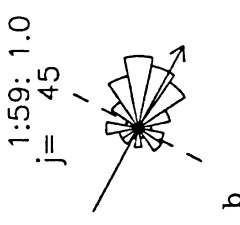

б

$5 \Xi$

ํㅡㅁ

芯 


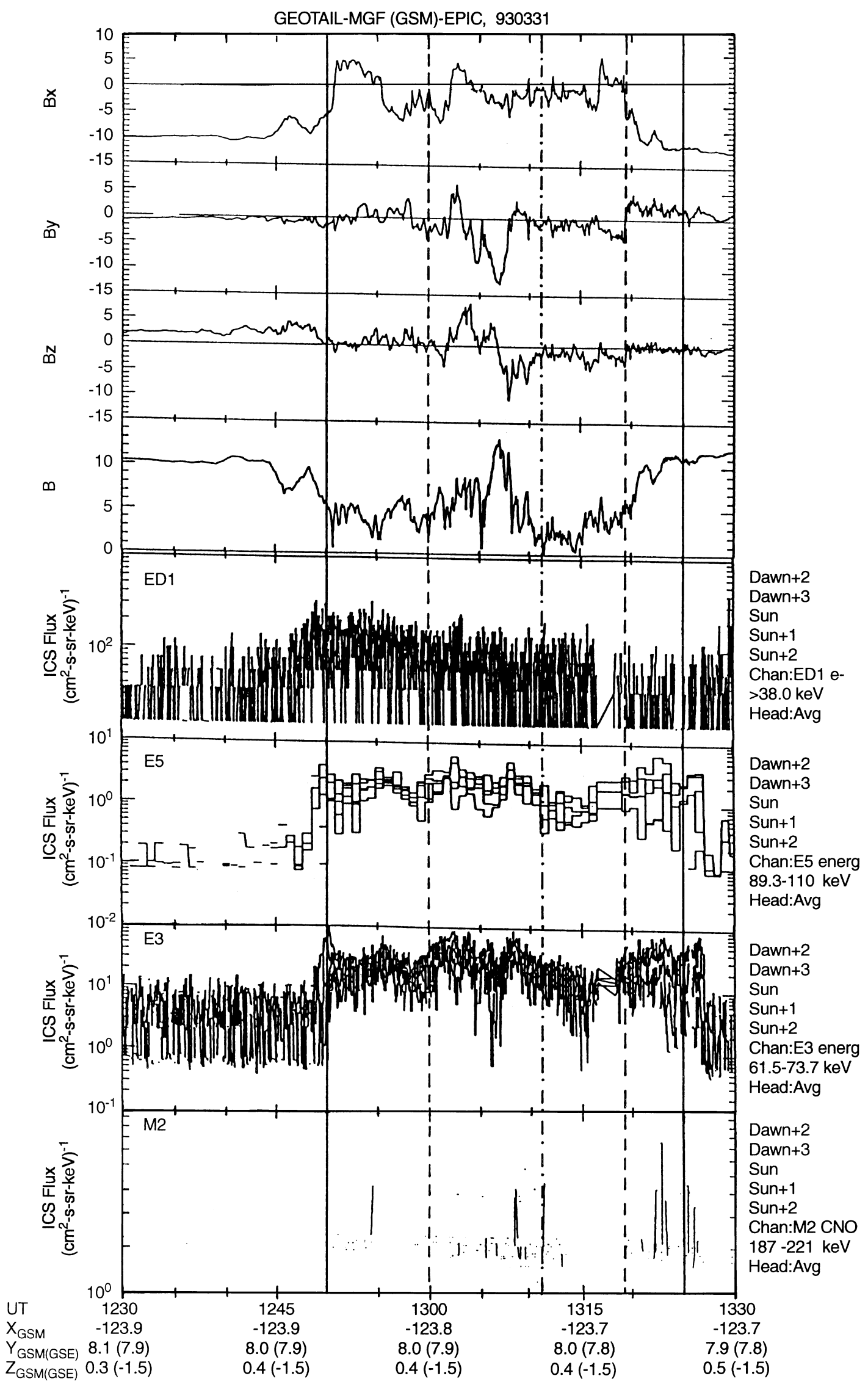

Fig. 5. An overview of the second particle event detected on March 31, 1993 at 1248 UT. The format is identical to the one in Fig. 1. The two solid vertical lines correspond to the two time periods 1250 UT and 1325 UT, when GEOTAIL leaves the lobe and returns to it

respectively. The two dashed vertical lines indicate the start (1300 UT) and the end times (1319 UT) of the convection interval. The Earthward edge of the bipolar Bz signature detected at $1311 \mathrm{UT}$ is marked by the vertical dashed-dotted line. 
show the three magnetic field components at $3 \mathrm{~s}$ resolution from the MGF experiment, together with the total magnetic field strength. The bipolar signature is along the Bz component. Just at the inflection point we observe a large By component which is due to the core field, thus the centre of this flux rope is aligned along the $\mathrm{Y}$ direction. The last four panels show the ICS flux of electrons (ED1 channel), all energy ions (E5 and E3 channel) and medium ions, mostly $\mathrm{O}^{+}$(M2 channel), from five sectors covering an angle of $112.5^{\circ}$, centred at the Sun $($ Dawn +2 , Dawn +3 , Sun, Sun +1 , Sun +2$)$.

The flux rope appeared at $\sim 1300$ UT at GEOTAIL location (this is the start time of convection, as will be determined in the following, using the angular distribution of ions), which is about $18 \mathrm{~min}$ after the substorm onset as seen in the AL index as a sharp decrease (marked in Fig. 2 with the second arrow), indicating a sudden increase in the westward electrojet.

As will be determined latter in the analysis of high resolution energetic particle, magnetic field and plasma data, the two solid vertical lines in Fig. 5, correspond to the two time epochs $1250 \mathrm{UT}$ and $1325 \mathrm{UT}$, when GEOTAIL leaves the lobe and returns to it respectively. The two dashed vertical lines indicate the start time (1300 UT) and the end time (1319 UT) of the convection interval. The earthward edge of the bipolar $\mathrm{Bz}$ signature detected at 1311 UT is marked in Fig. 5, by the vertical dashed-dotted line.

The proton and electron number densities, temperatures and bulk flow are displayed in Fig. 6. The magnetotail lobe was encountered from 1230 UT to $\sim 1250$ UT and from 1320 UT to 1350 UT. In the lobe the electron and proton temperatures were less than $10^{6} \mathrm{~K}$ and the tailward flow speed was less than $100 \mathrm{~km} /$ s. At 1245 UT, while the spacecraft was in the high-field south lobe hot earthward flowing plasmas were encountered (Fig. 6), with a bulk speed of $\sim 100 \mathrm{~km} / \mathrm{s}$. This layer of hot Earthward plasmas produced a noticeable deviation in the three components of the magnetic field, as seen in Fig. 5 .

The onset of the burst occurred right after the passage of this layer, and was characterized by a small isotropic increase in electron intensity at 1248:38 UT (Fig. 5). To determine more accurately the magnetospheric area where GEOTAIL was, we present, in Fig. 7, the angular distribution of the ions detected by E3 channel. The Earthward flowing particles are seen clearly in the first two distributions taken at 1245:25 UT and 1245:55 UT. Consequently at 1247:25 UT and 1248:02 UT a gradient anisotropy almost perpendicular to the field is observed, typical of ions at the edge of the PSBL, but still in the lobe (Cowley et al., 1984; Richardson and Cowley, 1985). The increase of electron counts is followed immediately by the dispersive onset of ions seen in Fig. 5.

From 1249 UT until 1251 UT the flow reversed to tailward, indicated also by a sharp negative peak on the Vx component of the bulk flow (see Fig. 6). Right after 1251 UT up to 1300 UT hot Earthward flowing plasmas were encountered as seen from the KP parameters of the

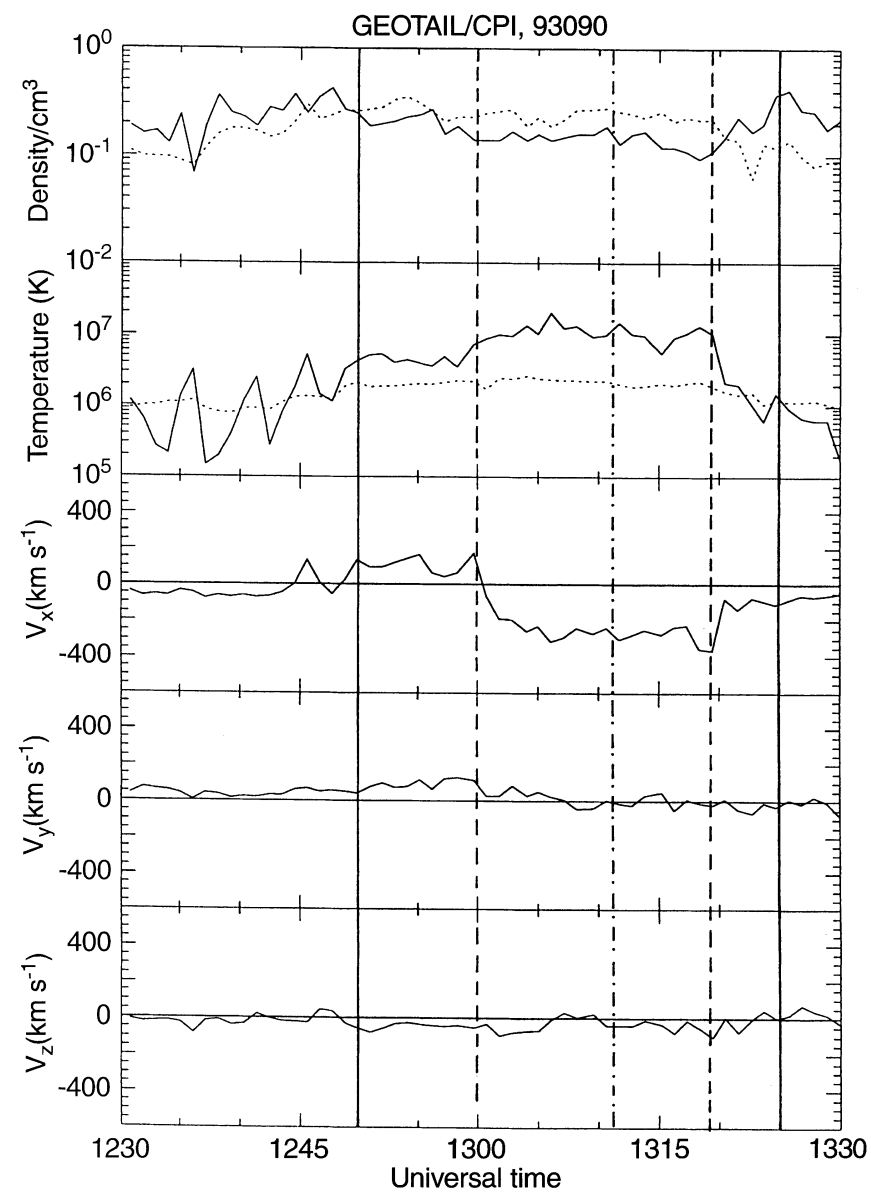

Fig. 6. The KP parameters of number densities and temperatures for protons (solid line) and electrons (dashed line) are shown in the upper two panels for the flux rope signature centred at 1307 UT on March 31, 1993. The components of proton bulk flow are shown in the lower three panels. The vertical lines are plotted as in Fig. 5.

CPI experiment. During this interval, the energetic particle flow looks isotropic (see Fig. 7, third row) with the maximum flow sometimes in the tailward direction and sometimes in the Earthward direction. This can be interpreted as the signature of two oppositely directed currents rather than statistical fluctuations in an isotropic distribution, since the counts during this interval are above background values. A north-to-south turning of the $B \mathrm{z}$ component from 1300 UT to $\sim 1311$ UT (Fig. 5), suggests the passage of the flux rope. The detailed study of the angular distribution of the ions, shown in Fig. 7, lead us to the conclusion that the ion population convected with the flux rope is observed from 1300 UT to 1319 UT. At the beginning of the convection interval, that is from 1300 UT to 1311 UT, GEOTAIL observes the north-to-south deviation in the $B \mathrm{z}$ component inside the plasma sheet. From 1311 UT until the end of the convection interval, at 1319 UT, GEOTAIL measures southward magnetic fields in the postplasmoid plasma sheet. After this time interval, the ions are still flowing tailward, but in this case the flow is aligned to the magnetic field, whereas during the convective interval the flow is always tailward independent of the magnetic field direction (see the two last 

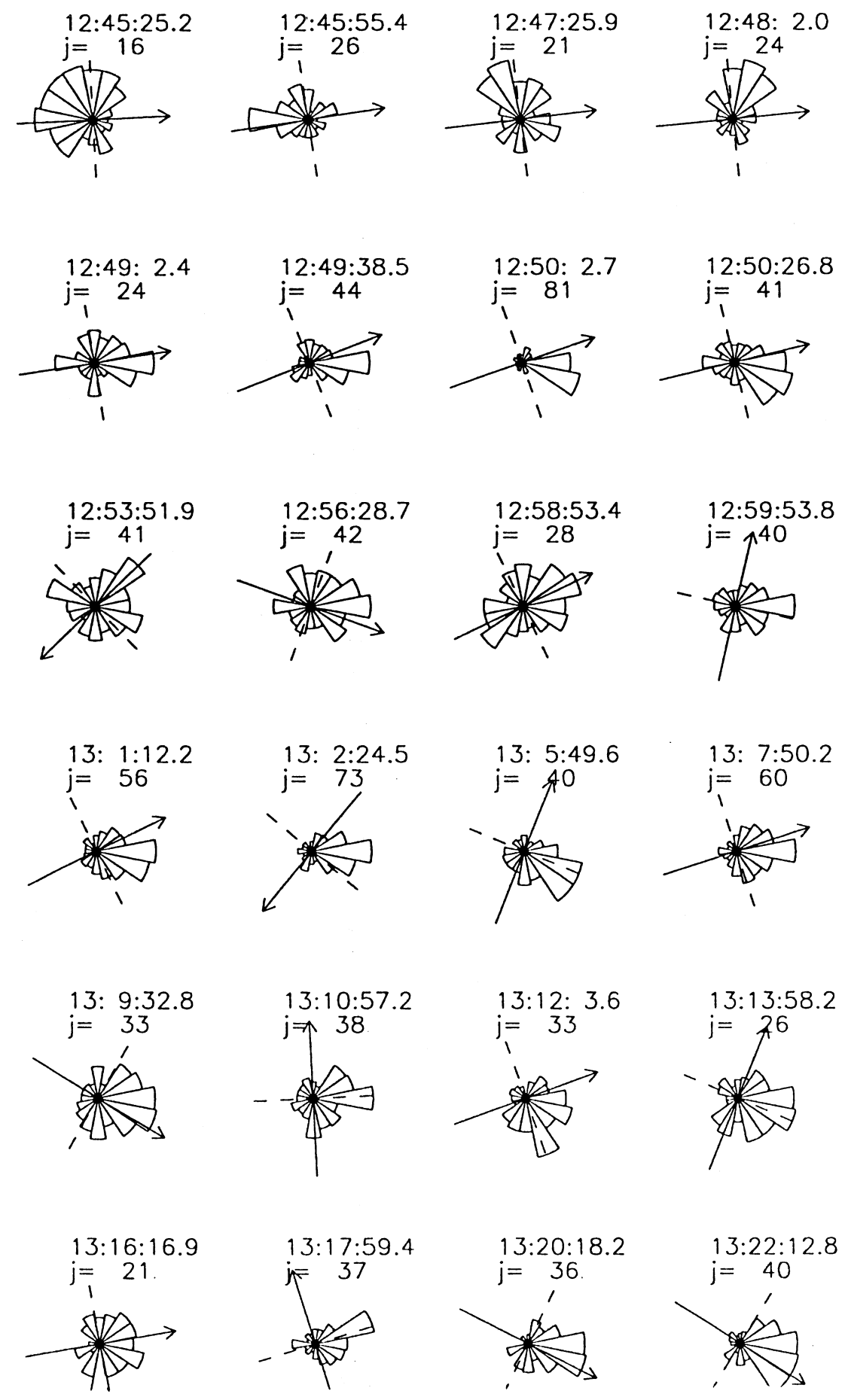

distributions in Fig. 7). The strong field-aligned tailward streaming ions are indicative of the PSBL crossing.

Given that GEOTAIL was at $\sim 124$ Re from the Earth, if we place the distant neutral line beyond this distance in its initial formation, the picture we get from the detailed description of this event is in accordance to the neutral line model of substorms, which is illustrated in Fig. 17 of Richardson and Cowley (1985). In this case GEOTAIL was initially located Earthward of the distant neutral line; therefore before the onset of the burst (i.e., the formation of the near-Earth neutral line) detects earthward flowing plasmas. Due to enhanced field line reconnection, the flux rope plasmoid and the quiet time neutral line are disconnected from the Earth and ejected tailward. At that time GEOTAIL detects tailward streaming particles. The sequence of events observed by GEOTAIL during this flux rope associated particle event, is given in Table 3.

The convective speed of the ion population moving with the magnetic structure may be estimated indirectly from the Compton-Getting effect (Gold et al., 1975). We found an anisotropy ratio of $\mathrm{J}_{\mathrm{f}} / \mathrm{J}_{\mathrm{b}}=3.3$ and a spectral 
Table 3. The sequence of events as determined from the analysis of GEOTAIL data for the flux rope signature centred at 1307 UT on March 31, 1993.

\begin{tabular}{lll}
\hline Time & Observation & GEOTAIL position \\
\hline 1242 UT & $\begin{array}{l}\text { Substorm onset on the ground } \\
\text { Increase of electrons }\end{array}$ & $\begin{array}{l}\text { South lobe } \\
\text { South lobe }\end{array}$ \\
1248 UT & $\begin{array}{l}\text { Velocity dispersion of energetic particles } \\
\text { 1249 UT to 1251 UT }\end{array}$ & PSBL \\
1251 UT to 1300 UT & $\begin{array}{l}\text { Hot Earthward flowing plasmas } \\
\text { Evidence of two oppositely directed } \\
\text { currents of energetic particles }\end{array}$ & \\
1300 UT to 1319 UT & $\begin{array}{l}\text { Convection } \\
\bullet \text { Bipolar Bz }\end{array}$ & • Plasma sheet \\
$\bullet$ 1300 UT to 1311 UT UT to 1319 UT & $\begin{array}{l}\text { - Southward Bz } \\
\text { S Post-plasmoid plasma sheet }\end{array}$ \\
\hline
\end{tabular}

index of $\gamma \sim 4.4$, which is consistent for this magnetospheric region. The estimated bulk speed according to this method is $200 \mathrm{~km} / \mathrm{s}$. The tailward bulk speed in the plasma sheet during the passage of the magnetic structure observed with the hot plasma analyser of the CPI varied from 200 to $250 \mathrm{~km} / \mathrm{s}$ during convection (see Fig. 6). Given that the high energy tail of the thermal ion population convected with the magnetic structure is observed for about $19 \mathrm{~min}$, the length of the magnetic structure based upon a mean convection speed from the plasma analyser of $\sim(300 \pm 60) \mathrm{km} / \mathrm{s}$ is $\sim(53 \pm$ 10) Re.

\section{c. Event of April 1, 1993 at 1330 UT}

The last event we will analyze in this study is an energetic particle burst that occurred on April 1, 1993 between 1325 UT and 1340 UT. Fig. 8 presents the overview of this event. GEOTAIL was located in the south lobe at $(\mathrm{X}, \mathrm{Y}, \mathrm{Z}) \approx(-117.7,4.5,-0.8) \mathrm{R}_{\mathrm{E}}$ in GSM co-ordinates. GEOTAIL was $\sim-3.5 \mathrm{R}_{\mathrm{E}}$ from the nominal aberrated axis, which means the spacecraft is on the dawn side. This event has a bipolar signature in both $B \mathrm{z}$ and $B y$ magnetic components with a strong core field primarily in the $\mathrm{x}$ direction. The magnetic field in the core of the structure exceeds the nearby lobe field by over $30 \%$. The inflection points of the two bipolar signatures are almost coincident, implying that the spacecraft passed through the centre of the structure. The beginning of the particle burst is marked by an increase of electrons at 1325 UT. Subsequently GEOTAIL detects an increase of ion fluxes, with small velocity dispersion.

The magnetic structure appeared at $\sim 1328$ UT at GEOTAIL's location as evidenced from the convection start time, determined by the particle distribution analysis that follows in the text, as well as from the start of bipolar signature in both $B y$ and $B z$ magnetic field components, marked in Fig. 8 by the first dashed vertical line. The second dashed vertical line indicates the end time (1338 UT) of the convection interval. The Earthward edge of the bipolar Bz signature detected at 1332 UT is marked in Fig. 8 by the vertical dasheddotted line.

This is also a substorm-associated event, as seen from the decrease of the AL index, presented in Fig. 9. The onset of the substorm on the ground occurred at $\sim 1310$ UT (marked in Fig. 9 by an arrow), that is 18 min prior to the plasmoid arrival at GEOTAIL.

The proton and electron densities and temperatures as well as the three components of the proton bulk speed from CPI experiment are presented in Fig. 10 from 1320 to $1343 \mathrm{UT}$, in $1 \mathrm{~min}$ resolution periods. According to the plasma parameters presented here, GEOTAIL is in the lobes from 1320 to 1325 UT and returns again after the passage of the structure at 1340 UT.

In the following we will analyze the detailed time history of this burst. The angular distributions of energetic electron $(\mathrm{E}>34 \mathrm{keV})$ and energetic ions (mainly protons) of energy $61 \mathrm{keV}<\mathrm{E}<73 \mathrm{keV}$, are presented in Fig. 11a and $\mathrm{b}$ respectively. The time shown at the top of the figure is the start time of the 6-s accumulation interval. The format of this figure is identical to that of Fig. 4. The tailward streaming direction is on the right. At the onset of the particle burst, while GEOTAIL is still in the south lobe, tailward streaming energetic electrons above background with a cross-tail component are observed for $1 \mathrm{~min}$, from 1324 UT to 1325 UT (see Fig. 11a). The angular distributions of ions presented in Fig. 11b show clearly the sequence of dynamic changes in the Earth's magnetotail during this burst. The first plot of this figure shows isotropic distribution, characteristic of the quiet lobe. The next two plots present tailward streaming ions with a gradient anisotropy perpendicular to the field, typical of ions at the edge of the PSBL but still in the lobe. The displacement of the PSBL across the spacecraft is obvious from the two distributions taken at 1325:25 UT and 1325:43 UT. A dawn-to-dusk component is superimposed on the tailward anisotropy. Convection starts as the structure passes over the spacecraft. The broader tailward angular distributions which indicate the convection mechanism starts at $\sim 1327$ UT, according to the plots presented in Fig. 9b. At the beginning of convection interval, from 1327 UT to 1332 UT GEOTAIL detects the plasma sheet magnetic field bipolar signature in both $B y$ and $B z$ components. For the rest of convection interval, until 1338 UT GEOTAIL detects the southward magnetic field of the post plasmoid plasma sheet (see Fig. 8). The spacecraft is found again in the PSBL at $\sim 1340$ UT, as shown by the strong tailward moving ions (Fig. 11b, last row). The sequence of events observed by GEOTAIL during this flux rope associated particle event, is given in Table 4. 


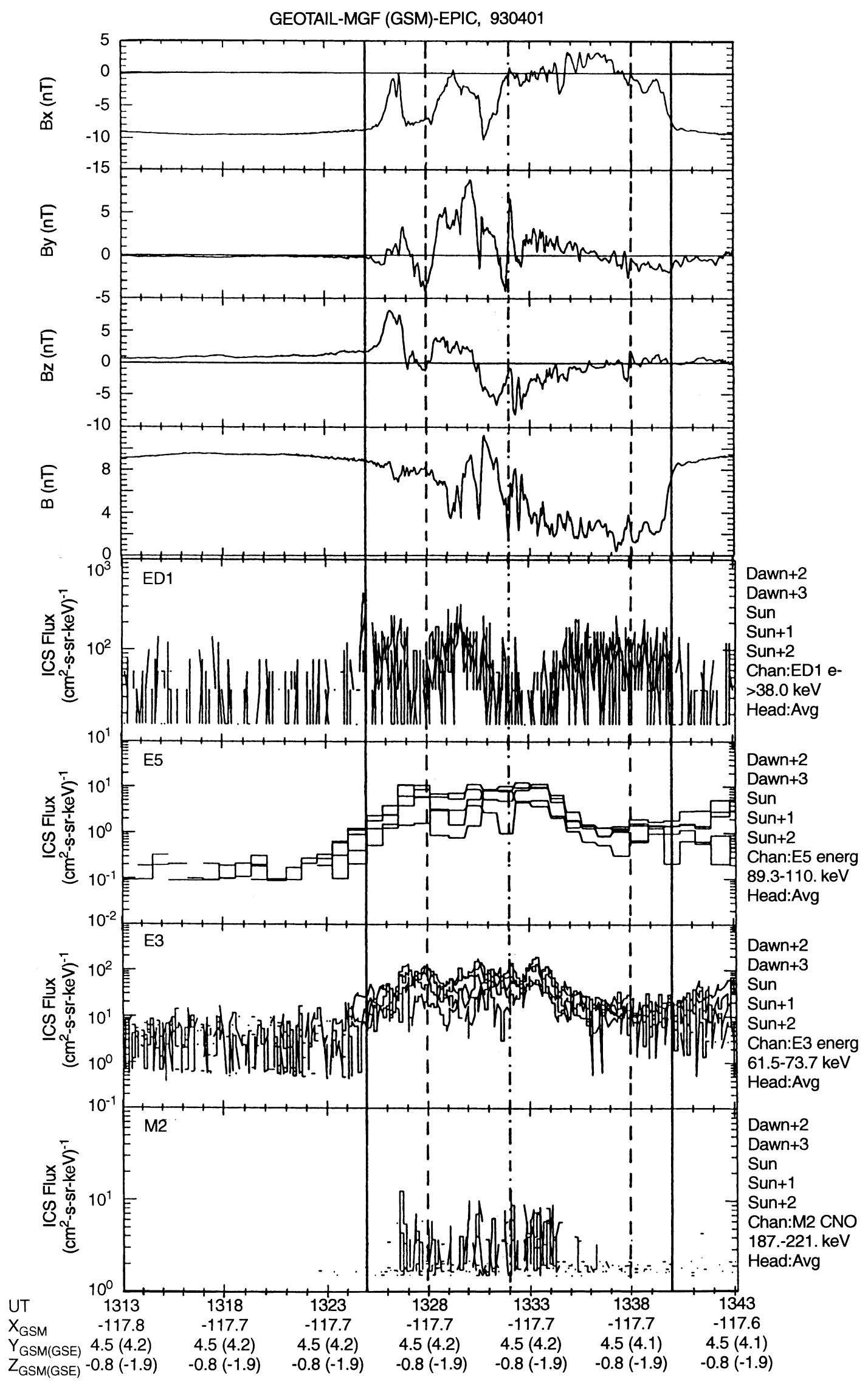

Fig. 8. An overview of the particle event on April 1, 1993 at 1324 UT. The format of this figure is identical to the one in Fig. 1. The two solid vertical lines correspond to the time periods 1325 UT and 1340 UT when GEOTAIL leaves the lobe and returns to it respectively. The

two dashed vertical lines indicate the start (1328 UT) and the end times (1338 UT) of convection interval. The Earthward edge of the bipolar $\mathrm{Bz}$ signature detected at $1332 \mathrm{UT}$ is marked by the vertical dasheddotted line. 

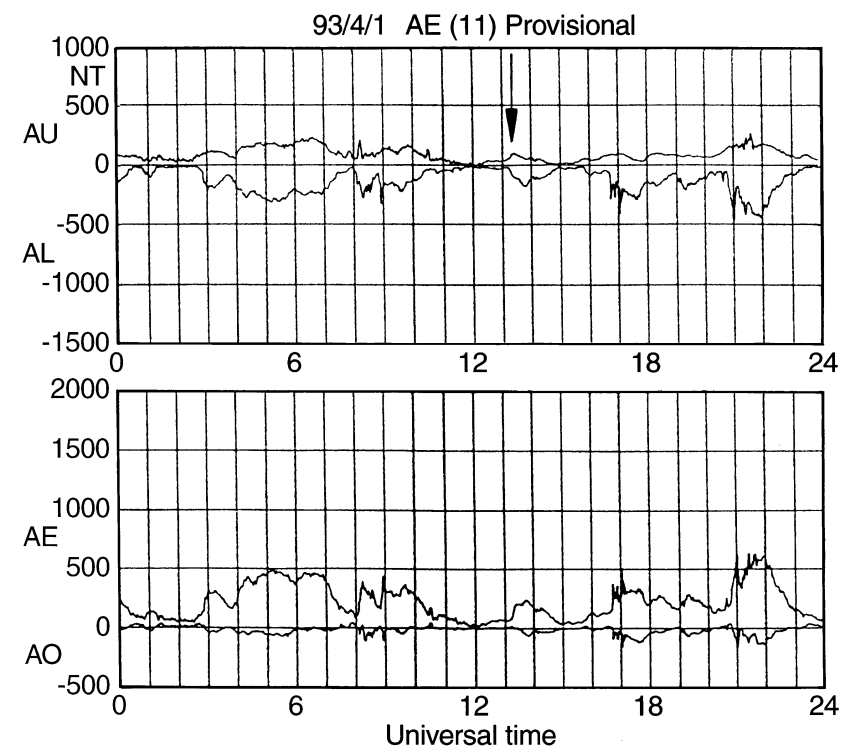

Fig. 9. The AE indices for 1 April 1993. The arrow indicates the onset of the substorm on the ground at $1310 \mathrm{UT}$, followed by the flux rope centred at 1331 UT.

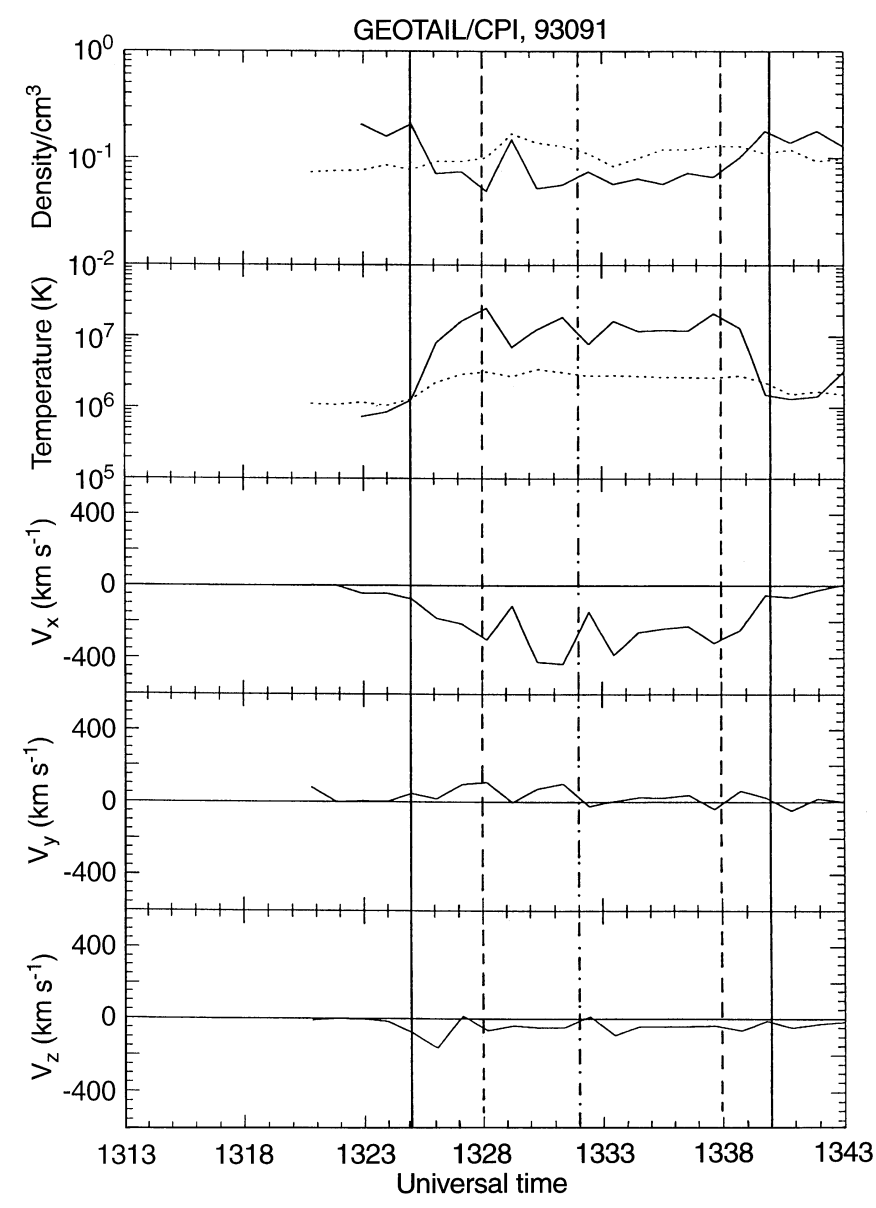

Fig. 10. The KP parameters of number densities and temperatures for protons (solid line) and electrons (dashed line) are shown in the upper two panels for the flux rope signature centred at 1331 UT on April 1, 1993. The components of proton bulk flow are shown in the lower three panels. The vertical lines are plotted as in Fig. 8.
The indirect estimation of the bulk speed from the Compton-Getting effect (Gold et al., 1975), gives a spectral index $\gamma \sim 4.1$ and an anisotropy ratio $\mathrm{j}_{\mathrm{f}} / \mathrm{j}_{\mathrm{b}}=10$, resulting in a bulk speed of $\approx 400 \mathrm{~km} / \mathrm{s}$. This is in agreement with the data obtained from the CPI experiment since the maximum tailward speed is $\sim 400 \mathrm{~km} / \mathrm{s}$ and it is detected when GEOTAIL crosses the core of the structure in the plasma sheet at 1331 UT. Given that the high energy tail of the thermal ion population convected with the magnetic structure is observed for about $10 \mathrm{~min}$, the length of the magnetic structure based upon a mean convection speed from the plasma analyser of $\sim(300 \pm 60) \mathrm{km} / \mathrm{s}$ is $\sim(28 \pm 5) \mathrm{Re}$.

\section{Discussion and conclusion}

We have studied three electron $(\geq 34 \mathrm{keV})$ and ion bursts $(62 \mathrm{keV} \leq \mathrm{E} \leq 110 \mathrm{keV})$ detected by GEOTAIL in the deep tail associated with the passage of a magnetic structure ejected downtail, all produced by isolated substorms. We reported several important features that appear to be consistent with the structure and evolution of the geomagnetic tail during substorms as predicted by the Hones $(1976,1977)$ model.

The start of the particle event at the spacecraft position (that may indicate the time of reconnection of the lobe field line the spacecraft is located on, but not the time of formation of the near-Earth neutral line, which may occur earlier) is marked by an increase of energetic electrons $(\mathrm{E}>38 \mathrm{keV})$, that quickly reach their maximum intensity, while the spacecraft is in the lobes, the area of open field lines. GEOTAIL detects the first energetic electrons $\sim 5-15 \mathrm{~min}$ after the onset of the expansion phase on the ground, as seen by the sudden decrease in the AL-index. This indicate a sudden increase of the westward electrojet, that marks the start of the unloading mechanism. The start of the particle event indicates the formation of the near-Earth neutral line earthward of GEOTAIL. The onset of energetic electrons is followed by an increase of the ion flux. The amount of dispersion observed in each one of the three events (velocity dispersion lasts from 3 to $7 \mathrm{~min}$ in the events presented here), depends on the transverse speed with which a layer of particles, spatially dispersed by speed, is pushed over the spacecraft by the advancing flux rope. In other words, velocity dispersion is associated with the displacement of the PSBL across the spacecraft. During this time interval, the ions show evidence of anisotropy associated with a gradient drift when strongly streaming in the plasma sheet boundary layer. Within the flux rope region, suprathermal ions exhibit a convective anisotropy, which allows determination of the plasma flow velocity, assuming that the anisotropy arises from the Compton Getting effect; the estimated convection speed varies between $200 \mathrm{~km} / \mathrm{s}$ and $650 \mathrm{~km} / \mathrm{s}$. This is in good agreement with the in situ observations of plasmas. While the speeds differ, this could simply arise because different events were studied. This speed is considerably less than that inferred from electron plasma and energetic ion observations with 


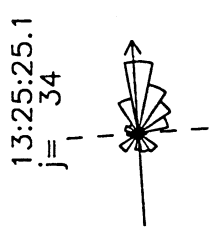

吕

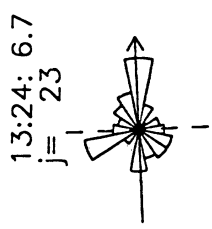

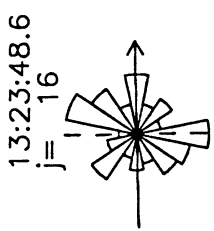

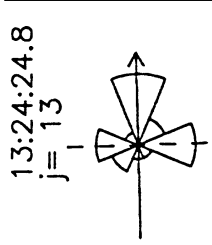

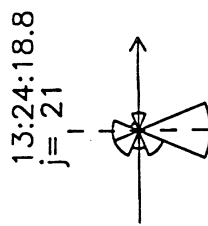

$\stackrel{\check{\dot{v}}}{\ddot{\tilde{n}}=}$

官的

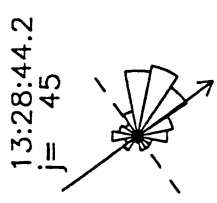

ஸ̃

官

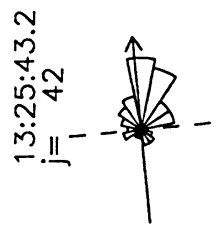

品-

i்
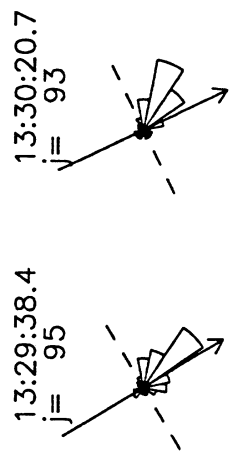

लें

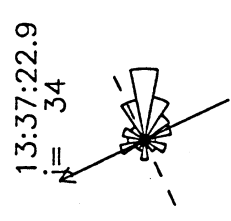

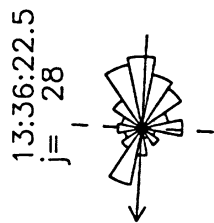

家.
ํㅛ

$\stackrel{\ddot{0}}{\ddot{0} \dot{m}}$

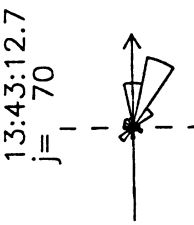

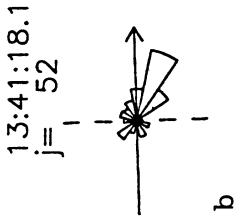

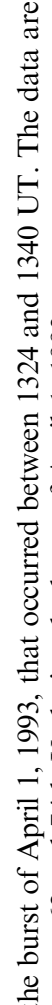

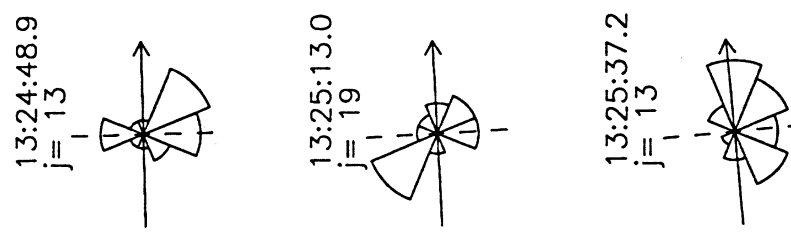

$\stackrel{m}{\ddot{m} \sigma}$

苛

$>0$

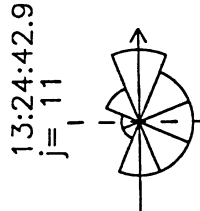

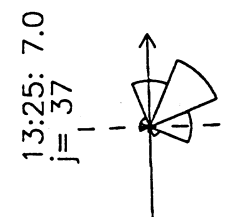

官

iัn

$\stackrel{\bar{m}}{\ddot{\ddot{n}}}=-1$
ํํำ

焉.

ஸ்.

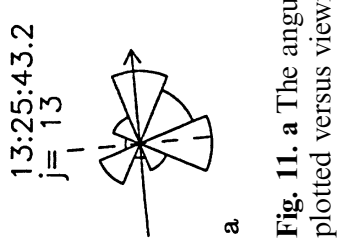


Table 4. The sequence of events as determined from the analysis of GEOTAIL data for the flux rope signature centred at 1331 UT on March 31, 1993.

\begin{tabular}{lll}
\hline Time & Observation & GEOTAIL position \\
\hline 1310 UT & Substorm onset on the ground & South lobe \\
1324 UT to 1325 UT & Tailward streaming electrons & South lobe \\
1325 UT to 1327 UT & $\begin{array}{l}\text { Velocity dispersion of energetic particles } \\
\text { 1327 UT to 1338 UT }\end{array}$ & $\begin{array}{l}\text { PSBL } \\
\bullet \text { Convection }\end{array}$ \\
$\bullet$ 1327 UT to 1332 UT & $\bullet$ Bipolar $B y$ and $B z$ & $\bullet$ Plasma sheet \\
\hline 1332 UT to 1338 UT & $\bullet$ Southward $B z$ & $\bullet$ Post-plasmoid plasma sheet \\
\hline
\end{tabular}

ISEE3 and timings derived from near-Earth and ground-based measurements, about 500 to $1000 \mathrm{~km} / \mathrm{s}$ (Hones et al., 1984; Baker et al., 1984; 1987; Scholer et al., 1984b). The magnetic field signature within the magnetic structure is a north-to-south turning of the $\mathrm{Bz}$ or/and $B y$ component, with the core field detected at the inflection point, exceeding the adjacent lobe field. Southward magnetic fields are observed in the postplasmoid plasma sheet earthward of flux rope as expected, due to continued reconnection of open lobe field lines, following the formation of the flux rope by reconnection primarily of closed plasma sheet field lines.

It is important to emphasize here that the moving magnetic structure interval is characterized by a convected ion population. This convective anisotropy is observed in the plasma sheet (bipolar signature in the $B \mathrm{z}$ or/and By component) and in the post-plasmoid plasma sheet (southward magnetic field). The length of the flux rope, based upon the duration of convection interval and the mean convection speed measured from the plasma analyser was found to be $\sim 56 \mathrm{Re}$ for the first two flux ropes and only $26 \mathrm{Re}$ for the last flux rope. Although half the size of the first two magnetic structures, it is within the normal range determined from previous plasmoid studies (Hones et al., 1984b; Richardson et al., 1987; Slavin et al., 1989). Although we cannot extract general conclusion from the analysis of only three events, it is interesting to note that the two larger flux ropes are associated with more intense isolated substorms. At the onset of the first substorm $\mathrm{AE}$ was $830 \mathrm{nT}$ whereas at the onset of the second substorm AE was $450 \mathrm{nT}$ (see Fig. 2). The smaller flux rope occurred on April 1, 1993 was associated with a weaker substorm, whose AE value at the onset of the expansion phase was only $200 \mathrm{nT}$. The suggestion, based upon these events, is that the amount of energy stored in the tail prior to substorm onset is proportional to the size of the released flux rope. Nevertheless, we have to note that the observed duration (and hence the inferred size) will also depend on the exact trajectory of the spacecraft through the structure. Therefore, one should not expect a simple correspondence between inferred size and the strength of the substorm.

Apart from these mentioned features which are consistent with the neutral line model, we can report also the following events.

Energetic electrons are enhanced sharply, rapidly before entering the plasma sheet and they decay rapidly before the PSBL crossing. On the other hand, energetic ions exhibit a different behaviour; they remain enhanced within the flux rope and in the PSBL when it is re-crossed after exit from the plasma sheet. The ions in the deep tail PSBL are observed when the flux rope arrives in the vicinity of the spacecraft and causes the PSBL to be swept out over the spacecraft. As originally proposed by Richardson et al. (1993) and Richardson et al. (1996), energetic electrons are accelerated rapidly at the substorm neutral line and escape rapidly down the tail. In contrast, ions with energies of tens of $\mathrm{keV}$ are accelerated along the current sheet so that their intensity remains high in the PSBL and the plasma sheet.

The study of the angular distributions of electrons and ions separately, in correlation with magnetic field observations leads us to some conclusions concerning the magnetic field topology of flux rope structures

In a more careful examination of the angular distributions of energetic electron with $\mathrm{E}>38 \mathrm{keV}$, presented in Figs. 4a and 11a, observed for some minutes after the onset of the burst, we can note that although the electrons are flowing in their background level fluxes, the distributions are not absolutely isotropic. We often observe cross-tail anisotropy and Earthward anisotropy. We have also to note the different behaviour of electrons and protons, observed with the hot plasma analyser of the comprehensive plasma instrumentation. During convection interval, the ion temperature increases by a factor of 20 , whereas the electron temperature increases by a factor of 2 , as shown in Figs 3, 6 and 10, where the convection interval is between the two vertical dashed lines. During the same time period, the ion density decreases, while the electron density remains more or less at the same level. Moreover, in a detailed study of the plasmoid signature centred at 1306 UT on March 31, 1993 (the second event presented here), Frank and Paterson (1994) have shown that the electron bulk flow velocity is tailward at about $500 \mathrm{~km} / \mathrm{s}$. Because the ion bulk speed is only about $200 \mathrm{~km} / \mathrm{s}$, also tailward, there must be an Earthward directed current associated with this magnetic signature. Thus, they suggested that the simple picture of co-moving ions and electrons associated with plasmoids or flux ropes is inadequate.

Furthermore, the existence of a strong magnetic field aligned approximately along the $\mathrm{Y}$-axis and centred on the north-to-south excursion of the field observed in each one of the three flux ropes, and the bipolar signature in both $B y$ and/or Bz components, distinguishes these magnetic structures from closed loops. All these features could argue against the picture of isolated magnetic structure and support the 3-D theoretical model of Hughes and Sibeck (1987), suggesting that closed field lines extend from Earth and wrap around the core. 
Acknowledgements. Thanks are due to Prof. L. A. Frank and to Dr W. R. Paterson for the CPI data provided.

Topical Editor K.-H. Glaßmeier thanks M. B. Moldwin and I.

G. Richardson for their help in evaluating this paper.

\section{References}

Baker, D. N., S. J. Bame, R. D. Belian, W. C. Feldman, J. T. Gosling, P. R. Higbie, E. W. Hones, Jr, D. J. McComas, and R. D. Zwickl, Correlated dynamical changes in the near-Earth and distant magnetotail regions: ISEE 3, J. Geophys. Res., 89, 3855, 1984.

Baker, D. N., R. C. Anderson, R. D. Zwickl, and J. A. Slavin, Average plasma and magnetic field variations in the distant magnetotail associated with near-Earth substorm effects, J. Geophys. Res., 92, 71-81, 1987.

Belehaki, A., E. T. Sarris, G. Tsiropoula, R. W. McEntire, S. Kokubun, T. Yamamoto, Energetic particle bursts detected by GEOTAIL in the distant tail, Proc. of the ICS-3, ESA SP389, 487-492, 1996a

Belehaki, A., E. T. Sarris, G. Tsiropoula, R. W. McEntire, S. Kokubun, T. Yamamoto, Flux rope signatures in the distant plasma sheet boundary layer observed by GEOTAIL: a case study, Proc. of the ICS-3, ESA SP-389, 507-510, 1996b.

Birn, J., M. Hesse, and K. Schindler, Filamentary structure of a three-dimensional plasmoid, J. Geophys. Res., 94, 241, 1989.

Cowley, S. W. H., R. J. Hynds, I. G. Richardson, P. W. Daly, T. R. Sanderson, K.-P. Wenzel, J. A. Slavin, and B. T. Tsurutani, Energetic ion regimes in the deep geomagnetic tail: ISEE3, Geophys. Res. Lett., 11, 275, 1984.

Coroniti, F. V., Explosive tail reconnection: the growth and expansion phases of magnetospheric substorms, J. Geophys. Res., 90, 7427, 1985.

Daly, P. W., T. R. Sanderson, and K.-P. Wenzel, Survey of energetic $(E>35 \mathrm{keV})$ ion anisotropies in the deep geomagnetic tail, J. Geophys. Res., 89, 10733, 1984.

Frank, L. A., and W. R. Paterson, Study of electron and ion bulk flows in the distant magnetotail with the GEOTAIL spacecraft, Gophys. Res. Lett., 21, 2963-2966, 1994.

Frank, L. A., K. L. Ackerson, W. R. Paterson, J. A. Lee, M. R. English, and G. L. Pickett, The comprehensive plasma instrumentation (CPI) for the Geotail spacecraft, J. Geomag. Geoelectr., 46, 23-37, 1994

Hones, E. W., Jr., The magnetotail: its generation and dissipation, Physics of Solar Planetary Environments, Ed. D. J. Williams, pp 557-571, AGU, Washington D.C., 1976.

Hones, E. W., Jr., Substorm processes in the magnetotail: comments on "On hot tenuous plasma, fireballs, and boundary layers in the Earth's magnetotail" L. A. Frank, K. L. Ackerson and R. P. Lepping, J. Geophys. Res., 82, 5633, 1977.

Hones, E. W., Jr, D. N. Baker, S. J. Bame, W. C. Feldman, J. T. Gosling, D. J. McComas, R. D. Zwickl, J. A. Slavin, E. J. Smith, and B. T. Tsurutani, Structure of the magnetotail at $220 \mathrm{R}_{\mathrm{E}}$ and its response to geomagnetic activity, Geophys. Res. Lett., 11, 5-7, 1984a.

Hones, E. W., Jr, J. Birn, D. N. Baker, S. J. Bame, W. C. Feldman, D. J. McComas, and R. D. Zwickl, Detailed examination of a plasmoid in the distant magnetotail with ISEE 3, Geophys. Res. Lett., 11, 1046, 1984b.

Hughes, W. J., and D. G. Sibeck, On the 3-dimensional structure of plasmoids, Geophys. Res. Lett. 14, 636-639, 1987

Gold, R. E., C. O. Bostrom, and E. C. Roelof, Anisotropy measurements of $\sim 50 \mathrm{keV}$ solar protons, Conf. Pap. Int. Cosmic Ray Conf. 14 $4^{\text {th }}, 5,1801,1975$.

Kokubun, S., T. Yamamoto, M. H. Acuna, K. Hayashi, K. Shiokawa, and H. Kawano, The GEOTAIL magnetic field experiment, J. Geomag. Geoelectr., 46, 7-21, 1994.
Lepping, R. P., J. A. Slavin, M. Hesse, J. A. Jones, and A. Szabo, Analysis of Magnetotail flux ropes with strong core fields: ISEE3 observations, J. Geomag. Geoelectr., 48, 589-601, 1996.

Lui, A. T. Y., D. J. Williams, S. P. Christon, R. W. McEntire, V. Angelopoulos, C. Jacquey, T. Yamamoto, and S. Kokubun, A preliminary assessment of energetic ion species in flux ropes/ plasmoids in the distant tail, Geophys. Res. Lett., 21, 3019-3022, 1994.

Maezawa, K., Magnetotail boundary motion associated with geomagnetic substorms, J. Geophys. Res., 80, 3543, 1975.

Moldwin, M. B., and W. J. Hughes, Plasmoids as magnetic flux ropes, J. Geophys. Res., 96, 14051-14064, 1991.

Moldwin, M. B., and W. J. Hughes, Plasmoid observations in the distant plasma sheet boundary layer, Geophys. Res. Lett., 19, 1911-1914, 1992a.

Moldwin, M. B., and W. J. Hughes, On the formation and evolution of plasmoids: a survey of ISEE3 geotail data, $J$. Geophys. Res., 97, 19259-19282, 1992 b.

Moldwin, M. B., and W. J. Hughes, Geomagnetic substorm association of plasmoids, J. Geophys. Res., 98, 81-88, 1993.

Murphy, N., J. A. Slavin, D. N. Baker, and W. J. Hughes, Enhancements of energetic ions associated with travelling compression regions in the deep geomagnetic tail, J. Geophys. Res., 92, 64-70, 1987.

Owen, C. J., and J. A. Slavin, Energetic ion events associated with traveling compression regions, in Proc. of the International Conference on Substorms -1, Eur. Space Agency Spec. Publ., ESA SP-000, 365-370, 1993.

Richardson, I. G., and S. W. H. Cowley, Plasmoid-associated energetic ion bursts in the deep geomagnetic tail: properties of the boundary layer, J. Geophys. Res., 90, 12133-12155, 1985.

Richardson, I. G., S. W. H. Cowley, E. W. Hones, Jr, and S. J. Bame, Plasmoid associated energetic ion bursts in the deep geomagnetic tail: properties of plasmoids and the postplasmoid plasma sheet, J. Geophys. Res., 92, 9997, 1987.

Richardson, I. G., C. J. Owen, J. A. Slavin, and T. T. von Rosenvinge, Energetic $(>0.2 \mathrm{MeV})$ electron bursts observed by ISEE 3 in the deep $(<240 \mathrm{Re})$ geomagnetic tail, J. Geophys. Res., 98, 13441-13451, 1993.

Richardson, I. G., C. J. Owen, and J. A. Slavin, Energetic $(>0.2 \mathrm{MeV})$ electron bursts in the deep geomagnetic tail observed by the Goddard Space Flight Center experiment on ISEE3: association with substorms, J. Geophys. Res., 101, 27232740, 1996.

Scholer, M., G. Gloeckler, B. Klecker, F. M. Ipavich, D. Hovestadt, and E. J. Smith, Fast moving plasma structures in the distant magnetotail, J. Geophys. Res., 89, 6717-6727, 1984a.

Scholer, M., G. Gloeckler, D. Hovestadt, B. Klecker, and F. M. Ipavich, Characteristics of plasmoidlike structures in the distant magnetotail, J. Geophys. Res., 89, 8872-8876, 1984b.

Scholer, M., D. Hovestadt, B. Klecker, G. Gloeckler, and F. M. Ipavich, Average flow between $70 \mathrm{Re}$ and $200 \mathrm{Re}$ in the geomagnetic tail, Geophys. Res. Lett., 11, 343, 1984c.

Sibeck, D. G., G. L. Siscoe, J. A. Slavin, E. J. Smith, S. J. Bame, and F. L. Scarf, Magnetotail flux ropes, Geophys. Res. Lett., 11, 1090-1093, 1984.

Slavin, J. A., E. J. Smith, B. T. Tsurutani, D. G. Sibeck, H. J. Singer, D. N. Baker, J. T. Gosling, E. W. Hones, and F. L. Scarf, Substorm associated traveling compression regions in the distant tail: ISEE-3 geotail observations, Geophys. Res. Lett., 11, 657-660, 1984.

Slavin, J. A., E. J. Smith, D. G. Sibeck, D. N. Baker, R. D. Zwickl, and S.-I. Akasofu, An ISEE3 study of average and substorm conditions in the distant magnetotail, J. Geophys. Res., 90, 10875-10895, 1985.

Slavin, J. A., D. N. Baker, J. D. Craven, R. C. Elphic, D. H. Fairfield, L. A. Frank, A. B. Galvin, H. J. Hughes, R. H. Manka, D. G. Mitchell, I. G. Richardson, T. R. Sanderson, D. G. Sibeck, E. J. Smith, and R. D. Zwickl, CDAW-8 observations of plasmoid signatures in the geomagnetic tail: an assessment, J. Geophys. Res., 94, 15153-15175, 1989. 
Slavin, J. A., M. F. Smith, E. L. Mazur, D. N. Baker, E. W. Hones, Jr., T. Iyemori, and E. W. Greenstadt, ISEE3 observations of travelling compression regions in the Earth's magnetotail, J. Geophys. Res., 98, 15425-15446, 1993.

Stern, D., The role of O-type neutral lines in magnetic merging during substorms and solar flares, J. Geophys. Res., 84, 63-71, 1979.

Taguchi, S., J. A. Slavin and R. P. Lepping, Traveling compression region observed in the mid-tail lobes near substorm expansion phase onset, Proc. Third International Conference on Substorms, ESA SP-389, 603-607, 1996.
Williams, D. J., R. W. McEntire, C. Schlemm II, A. T. Y. Lui, G. Gloeckler, S. P. Christon, and F. Gliem, GEOTAIL energetic particles and ion composition instrument, J. Geomag. Geoelectr. 46, 39-57, 1994.

Zwickl, R. D., D. N. Baker, S. J. Bame, W. C. Feldman, J. T. Gosling, E. W. Hones, Jr., and D. J. McComas, Evolution of the Earth's distant magnetotail: ISEE3 electron plasma results, J. Geophys. Res., 89, 11007-11012, 1984. 